\title{
An asparagine oxygenase (AsnO) and a 3-hydroxyasparaginyl phosphotransferase (HasP) are involved in the biosynthesis of calcium-dependent lipopeptide antibiotics
}

Correspondence

Jason Micklefield

jason.micklefield@manchester. ac.uk

Received 21 September 2006

Revised 7 November 2006

Accepted 17 November 2006

\author{
Joanne M. Neary, ${ }^{1,2}$ Amanda Powell, ${ }^{1}$ Lyndsey Gordon, ${ }^{1,2}$ Claire Milne, ${ }^{1}$ \\ Fiona Flett, ${ }^{2}$ Barrie Wilkinson, ${ }^{3}$ Colin P. Smith $^{2} \dagger$ and Jason Micklefield ${ }^{1} \ddagger$
'School of Chemistry, The University of Manchester, PO Box 88, Manchester M60 1OD, UK CB10 1XL, UK \\ ${ }^{2}$ Department of Biomolecular Sciences, UMIST, PO Box 88, Manchester M60 1QD, UK \\ ${ }^{3}$ Biotica Technology Ltd, Chesterford Research Park, Little Chesterford, Saffron Walden, Essex
}

\begin{abstract}
Nonribosomal peptides contain a wide range of unusual non-proteinogenic amino acid residues. As a result, these complex natural products are amongst the most structurally diverse secondary metabolites in nature, and possess a broad spectrum of biological activities. $\beta$-Hydroxylation of amino acid precursors or peptidyl residues and their subsequent processing by downstream tailoring enzymes are some of the most common themes in the biosynthetic diversification of these therapeutically important peptides. Identification and characterization of the biosynthetic intermediates and enzymes involved in these processes are thus pivotal in understanding nonribosomal peptide assembly and modification. To this end, the putative asparaginyl oxygenase- and 3-hydroxyasparaginyl phosphotransferase-encoding genes has $P$ and asn $O$ were separately deleted from the calcium-dependent antibiotic (CDA) biosynthetic gene cluster of Streptomyces coelicolor. Whilst the parent strains produce a number of 3-hydroxyasparagine- and 3-phosphohydroxyasparagine-containing CDAs, the $\triangle$ has $P$ mutants produce exclusively non-phosphorylated CDAs. On the other hand, $\triangle a s n O$ mutants produce several new Asn-containing CDAs not present in the wild-type, which retain calcium-dependent antimicrobial activity. This confirms that $\mathrm{AsnO}$ and $\mathrm{HasP}$ are required for the $\beta$-hydroxylation and phosphorylation of the Asn residue within CDA.
\end{abstract}

\section{INTRODUCTION}

The calcium-dependent antibiotics (CDAs) from Streptomyces coelicolor (Hojati et al., 2002; Kempter et al., 1997) belong to the group of structurally related acidic

†Present address: School of Biomedical and Molecular Sciences, University of Surrey, Guildford GU2 7XH, UK.

łPresent address: Manchester Interdisciplinary Biocentre, The University of Manchester, 131 Princess Street, Manchester M1 7DN, UK.

Abbreviations: CAS, clavaminic acid synthase; CDA, calcium-dependent antibiotic; D-HPG, D-hydroxyphenylglycine; EGF, epidermal growth factor; ESI, electrospray ionization; LC, liquid chromatography; 3MeGlu, L-3-methylglutamic acid; mw, molecular weight; 3-OHAsn, (2R,3S)-3-hydroxyasparagine; 3-OPAsn, (2R,3S)-3-phosphohydroxyasparagine; PCP, peptidyl carrier protein.

Tables detailing the bacterial strains, plasmids, primers and PCR products used in this work, and also a figure showing the PCR analysis of $\Delta a s n O$ and $\Delta$ has $P$ mutants, are available as supplementary data with the online version of this paper. lipopetide antibiotics, which include A54145 (Fukuda et al., 1990; Miao et al., 2006), daptomycin (Baltz et al., 2005; Miao et al., 2005; Raja et al., 2003), friulimicins and amphomycins (Vértesy et al., 2000). All of these nonribosomally biosynthesized lipopeptides contain $N$-terminal fatty acid side chains, which is a trans-2,3-epoxyhexanoyl moiety in the case of CDA (Fig. 1), along with decapeptide lactone or lactam cores. Within the decapeptide cores are a number of conserved amino acids, including acidic residues responsible for co-ordination of calcium ions, which are essential for antimicrobial activity. Notably, daptomycin was recently approved for clinical use, becoming the first new structural class of natural antibiotic to reach the clinic in over 30 years (Baltz et al., 2005; Raja et al., 2003). As a result of this, there has been considerable interest in establishing the biosynthetic origins of the acidic lipopeptide group of antibiotics, with the specific aim of engineering new lipopeptide variants with improved therapeutic properties. To this end we have focused on engineering the biosynthesis of CDAs, using adenylation domain active site modifications to replace Asp 


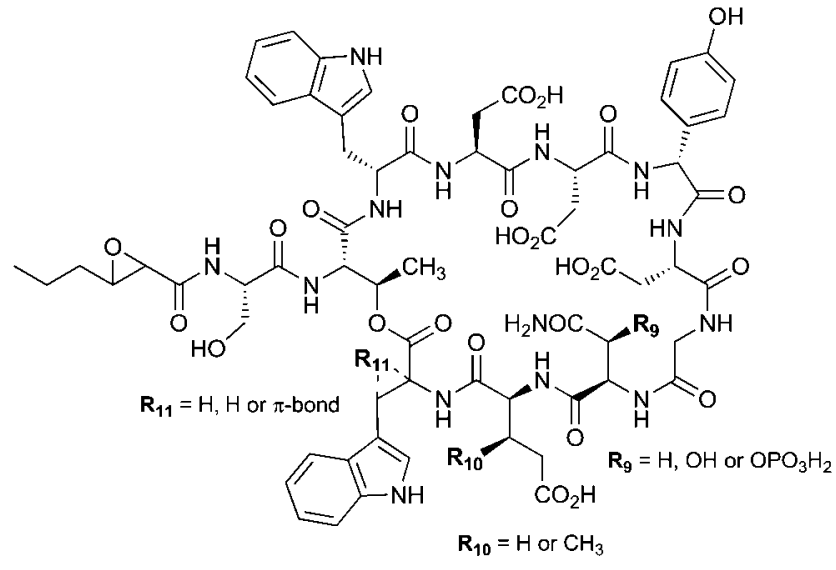

\begin{tabular}{|lccccl}
\hline CDAx & $\mathbf{R} \mathbf{9}$ & $\mathbf{R}_{10}$ & $\mathbf{R}_{11}$ & $\boldsymbol{m} \boldsymbol{w}$ & \multicolumn{1}{c}{ Reference } \\
\hline CDA1b & $\mathrm{OPO}_{3} \mathrm{H}_{2}$ & $\mathrm{H}$ & $\mathrm{H}, \mathrm{H}$ & 1562.5 & Kempter et al. (1997) \\
CDA2b & $\mathrm{OPO}_{3} \mathrm{H}_{2}$ & $\mathrm{CH}_{3}$ & $\mathrm{H}, \mathrm{H}$ & 1576.5 & Kempter et al. (1997) \\
CDA3b & $\mathrm{OH}$ & $\mathrm{H}$ & $\mathrm{H}, \mathrm{H}$ & 1482.5 & Kempter et al. (1997) \\
CDA4b & $\mathrm{OH}$ & $\mathrm{CH}_{3}$ & $\mathrm{H}, \mathrm{H}$ & 1496.5 & Kempter et al. (1997) \\
CDA2a & $\mathrm{OPO} \mathrm{H}_{2}$ & $\mathrm{CH}_{3}$ & $\pi$-bond & 1574.5 & Hojati et al. (2002) \\
CDA3a & $\mathrm{OH}$ & $\mathrm{H}$ & $\pi$-bond & 1480.5 & Hojati et al. (2002) \\
CDA4a & $\mathrm{OH}$ & $\mathrm{CH}_{3}$ & $\pi$-bond & 1494.5 & Hojati et al. (2002) \\
CDA5a & $\mathrm{H}$ & $\mathrm{H}$ & $\pi$-bond & 1464.5 & This work \\
CDA6a & $\mathrm{H}$ & $\mathrm{CH}_{3}$ & $\pi$-bond & 1478.5 & This work \\
\hline
\end{tabular}

Fig. 1. Structures of CDA variants. The $b$ series CDAs contain L-Trp at position 11, whilst the a series possess Z-dehydrotryptophan residues at position 11 (Hojati et al., 2002; Kempter et al., 1997). CDA5a and $6 a$ are novel biosynthetically engineered CDAs possessing Asn at position 9.

at position 7 in CDAs with Asn (Uguru et al., 2004) and a mutasynthesis approach to replace the D-hydroxyphenylglycine (D-HPG) at position 6 with alternative arylglycines (Hojati et al., 2002).

In addition to D-HPG, CDA contains a number of other non-proteinogenic amino acids, including L-3-methylglutamic acid (3-MeGlu; at position 10), which is also present at the same relative position in the decapeptide cores of daptomycin and A54145 (Milne et al., 2006), and a Cterminal Z-dehydrotryptophan residue (Hojati et al., 2002). CDA variants which contain either $(2 R, 3 S)$-3-hydroxyasparagine (3-OHAsn) or (2R,3S)-3-phosphohydroxyasparagine (3-OPAsn) at position 9 are also known. Whilst 3 -OHAsn is common to a number of peptide antibiotics, including A54145 (Fukuda et al., 1990; Miao et al., 2006), katanosin B (Kato et al., 1988) and ramoplanin (Walker et al., 2005; McCafferty et al., 2002), 3-phosphohydroxyasparagine has not been found elsewhere in nature, to date.

Previously, we analysed the $c d a$ biosynthetic gene cluster and identified a gene, SCO3236, which encodes a protein that shows high similarity to clavaminate synthase (CAS), an $\mathrm{Fe}(\mathrm{II}) / 2$-oxoglutarate-dependent oxygenase from
Streptomyces clavuligerus. CAS catalyses the $\beta$-hydroxylation of the Arg side chain of deoxyguanidinoproclavaminic acid, and subsequent cyclization and dehydrogenation steps during the biosynthesis of clavulanic acid (Baldwin et al., 1993; Salowe et al., 1990). On this basis, we suggested that SCO3236 encodes an asparagine oxygenase (AsnO) that was predicted to catalyse the $\beta$-hydroxylation of an Asn residue in a CDA precursor to generate the 3-OHAsn found in CDA (3a, 3b, $4 \mathrm{a}$ and $4 \mathrm{~b}$ variants) (Fig. 1).

$\beta$-Hydroxylation of amino acids is a common phenomenon in the biosynthesis of nonribosomal peptides (Chen et al., 2001, 2002). Indeed the subsequent processing of the amino acid $\beta$-hydroxyl groups by further oxidation (Chen et al., 2002), glycosylation (Lu et al., 2004), macrolactonization (Kato et al., 1988; Walker et al., 2005; McCafferty et al., 2002), methylation (Miao et al., 2006) or in the case of CDA, phosphorylation, serves to increase the structural diversity of nonribosomal peptides and related products, resulting in a wide range of biological activities. Of the many $\beta$ hydroxylated amino acids and derivatives found in nonribosomal peptides, some arise as a result of the oxidation of free amino acids prior to peptide assembly (Yin \& Zabriskie, 2004; Ju et al., 2004; Haltli et al., 2005). For example, in the biosynthesis of viomycin and mannopeptimycin, L-Arg and the non-proteinogenic amino acid L-enduracididine are hydroxylated as free amino acids by non-haem $\mathrm{Fe}(\mathrm{II}) / 2$ oxoglutarate-dependent oxygenases VioC (Yin \& Zabriskie, 2004; Ju et al., 2004) and MppO (Haltli et al., 2005), respectively. In addition, there are a number of other nonhaem $\mathrm{Fe}(\mathrm{II}) / 2$-oxoglutarate-dependent oxygenases which are known to hydroxylate specific amino acid residues within ribosomal polypeptides. For example, mammalian asparaginyl hydroxylases EGFH and FIH are known, which catalyse the posttranslational $\beta$-hydroxylation of Asn residues within epidermal growth factor (EGF) domains (Jia et al., 1994) and the hypoxia-inducible factor (HIF) Cterminal transactivation domains (Hewitson et al., 2002). Finally, several monooxygenase enzymes have been characterized that function on amino acid thioesters that are tethered to carrier proteins via flexible phosphopantetheine prosthetic groups (Chen et al., 2001, 2002). These include haem protein hydroxylases (ORF20, NovI and NikQ), which are responsible for the $\beta$-hydroxylation of peptidyl carrier protein (PCP)-tethered tyrosine and histidine residues during the biosynthesis of chloroeremomycin, novobiocin (Chen et al., 2001) and nikkomycin (Chen et al., 2002).

Downstream of $a s n O$, in the CDA gene cluster, is a gene, SCO3234, which encodes a protein that shows sequence similarity to a number of ATP-dependent aminoglycoside phosphotransferases, including $\mathrm{SpcN}$, which has been shown to phosphorylate spectinomycin as part of the host self-resistance mechanism in the producer strain Streptomyces flavopersicus (Lyutzkanova et al., 1997). Based on this, we suggested that the SCO3234 gene product is a putative 3-hydroxyasparagine phosphotransferase (HasP), which phosphorylates the 3-hydroxyasparaginyl 
residue of $\mathrm{CDA} 3 \mathrm{a} / \mathrm{b}$ and $\mathrm{CDA} 4 \mathrm{a} / \mathrm{b}$ to produce the 3phosphohydroxyasparaginyl residues found in CDA1b and CDA2a/b (Fig. 1). In this paper, we aim to determine the in vivo function of the asn $\mathrm{O}$ and hasP gene products, and test the hypothesis that these products are involved in the hydroxylation and subsequent phosphorylation of the asparaginyl residues in CDA. It was envisaged that this insight would provide a means to control the functionality of the Asn-derived residues in CDA which could be further utilized to engineer other therapeutically relevant acidic lipopeptides based around the A54145 and daptomycin biosynthetic templates.

\section{METHODS}

Bacterial strains, plasmids and culture conditions. The bacterial strains and plasmids used in this study are listed in Supplementary Table S1. Escherichia coli strains were propagated routinely in Luria-Bertani (LB) broth or on agar plates at $37^{\circ} \mathrm{C}$. Antibiotics were used in selective media at the standard concentrations (Sambrook et al., 2000). For DNA manipulation, Streptomyces strains were routinely propagated in YEME liquid media or on R2YE agar plates at $30^{\circ} \mathrm{C}$ (Kieser et al., 2000).

DNA preparation, manipulation and sequencing. S. coelicolor cosmid ScE29 encompassing SCO3232-SCO3249 of the CDA biosynthetic gene cluster was obtained from the John Innes Centre, Norwich, UK, and was isolated from E. coli using the Qiagen Midiprep Kit. Small-scale plasmid preparation was carried out using the alkaline lysis method (Birnboim \& Doly, 1979), unless a higher quality of DNA was required for sequencing, in which case DNA was prepared using a Midiprep Kit. Streptomyces genomic DNA was prepared as described elsewhere (Kieser et al., 2000). Restriction enzymes were purchased from Roche, New England Biolabs and MBI Fermentas. Alkaline phosphatase, T4 DNA ligase and Pwo polymerase were all purchased from Roche. All enzymes were used according to the manufacturers' recommendations, unless otherwise specified.

PCR amplification was carried out on a GeneAmp PCR system 2400 (Perkin-Elmer) using 100 pmol of each primer, $\sim 100 \mathrm{ng}$ cosmid or 200 ng genomic DNA template per $100 \mu$ reaction, and 1 unit of the high fidelity Pwo polymerase. PCR reactions also contained 5\% (v/v) DMSO to overcome potential secondary structure formation in the GC-rich Streptomyces DNA. Following amplification, PCR products were purified using the Qiaquick extraction kit (Qiagen). PCR products and primers used in this work are detailed in
Supplementary Table S2. DNA sequencing of PCR products was carried out by the chain-termination method using the ABI/PRISM dye terminator cycle sequencing core kit (Perkin-Elmer). E. coli plasmid construction and transformations where carried out using standard procedures (Sambrook et al., 2000).

Construction of the asno deletion cassette. An upstream region of SCO3236 was PCR-amplified using primers AsnOF2 and AsnOR2, containing XhoI and PstI restriction sites, respectively (Supplementary Table S2), with ScE29 as a template to generate a $1558 \mathrm{bp}$ amplicon (fragment A) containing the first $226 \mathrm{bp}$ of SCO3236. Fragment A and pMT3000 (Paget et al., 1994) were digested with XhoI and PstI, and ligated to form plasmid pJN9a. Primers AsnOF3 and AsnOR3, containing EcoRI and XhoI restriction sites, were used in a PCR reaction with ScE29 as a template to generate a $1662 \mathrm{bp}$ amplicon (fragment B) containing the last $227 \mathrm{bp}$ of SCO3236. Fragment B and pMT3000 were digested with EcoRI and $X h o I$, and ligated to form pJN9b. Fragment A was excised from $\mathrm{pJN} 9 \mathrm{a}$ via $P s t \mathrm{I} / \mathrm{XhoI}$ and ligated into similarly digested $\mathrm{pJN} 9 \mathrm{~b}$ to give the plasmid pJN10 containing the $\operatorname{asnO}(\mathrm{AB})$ deletion construct (Fig. 2). This was excised from pJN10 via the flanking BglII sites and ligated into the BamHI site of pKC1132 (Bierman et al., 1992), resulting in $\mathrm{pJN} 12$, which was used for delivery of the truncated asnO into the chromosome of S. coelicolor 2377 (Hopwood \& Wright, 1983) and MT1110 (Hindle \& Smith, 1994) strains.

Construction of the hasP deletion cassette. An upstream region of SCO3234 was PCR-amplified using primers HasPF1 and HasPR1, containing PstI and $\mathrm{XbaI}$ restriction sites, respectively, with ScE29 as a template to generate a $1830 \mathrm{bp}$ amplicon (fragment $\mathrm{A}^{\prime}$ ) containing the first $70 \mathrm{bp}$ of SCO3234. Fragment $\mathrm{A}^{\prime}$ and pMT3000 were digested with PstI and XbaI, and ligated to form pMUT1. Primers HasPF2 and HasPR2, containing XbaI and XhoI restriction sites, respectively, and the template ScE29 were used in the PCR amplification of a $1939 \mathrm{bp}$ amplicon (fragment $\mathrm{B}^{\prime}$ ) containing the last 215 bp of SCO3234. Fragment B' and pMT3000 were digested with $X b a \mathrm{I}$ and $X h o \mathrm{I}$, and ligated to form pMUT2, excised from pMUT2 via $X b a \mathrm{I} / \mathrm{XhoI}$, and ligated into similarly digested pMUT1 to give the plasmid pLG3 containing the hasP deletion construct $\left(\mathrm{A}^{\prime} \mathrm{B}^{\prime}\right)$ (Fig. 2). The deletion construct was excised from pLG3 via the flanking BglII sites and ligated into the BamHI site of pMAH (Paget et al., 1994), resulting in pLG4, which was used for delivery of the truncated hasP into the chromosome of S. coelicolor 2377 and MT1110 strains.

Deletion of asnO and hasP from S. coelicolor MT1110 and 2377. $\triangle a s n O$ and $\triangle$ has $P$ delivery plasmids pJN12 and pLG4 were first passed through E. coli strain ET12567 to remove Dam/Dcm methylation. The plasmids were then alkaline-denatured as described elsewhere (Oh \& Chater, 1997) and introduced into Streptomyces

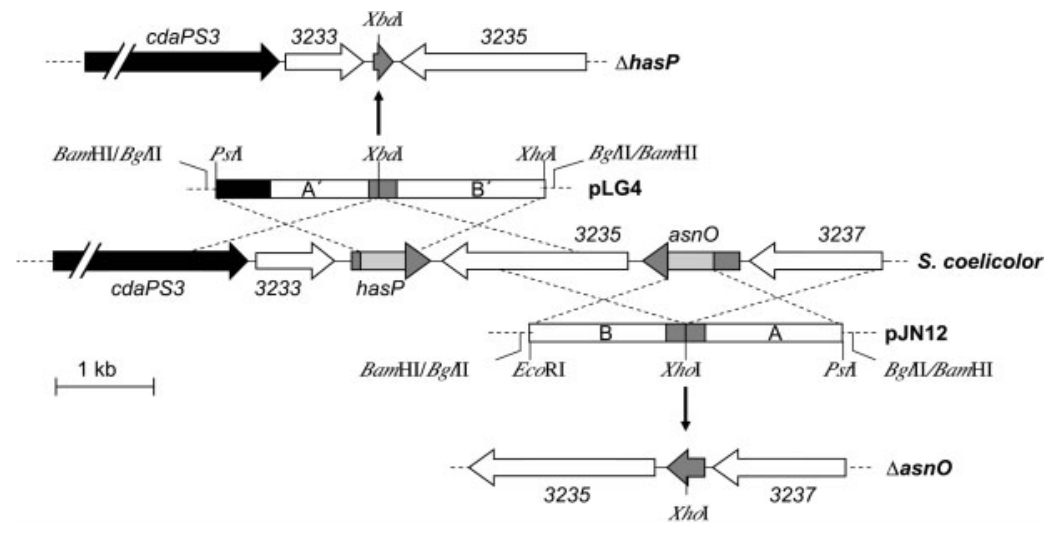

Fig. 2. Generation of $S$. coelicolor $\Delta a s n O$ and $\Delta$ has $P$ mutant strains. Plasmids pLG4 and pJN12, which contain truncated has $P$ $\left(\mathrm{A}^{\prime} \mathrm{B}^{\prime}\right)$ and asnO $(\mathrm{AB})$ deletion constructs, respectively, were used to perform standard double-crossover gene replacements, which resulted in mutants possessing in-frame truncated has $P$ and asn $O$ genes, respectively. 
protoplasts using the PEG-mediated transformation method (Kieser et al., 2000). Primary integrants of pJN12 and pLG4 were selected for with apramycin and hygromycin, respectively, and grown for two generations on non-selective agar to allow double-crossover events to occur. Colonies were screened for the loss of antibiotic resistance by replica plating. Total genomic DNA was isolated from antibiotic-sensitive recombinants, and used as a template for PCR amplification using primers flanking the deleted region. Primers $29.05 \mathrm{seqF} 2$ and $29.05 \mathrm{R} 2$ amplified the region of $a s n O$, giving products of size $1044 \mathrm{bp}$ from strains that had not undergone gene replacement and $495 \mathrm{bp}$ from strains that had a truncated form of asnO (Supplementary Fig. 1A). Similarly, primers HasPKO1 and HasPKO2 were used to amplify the region of hasP, resulting in products of size $1818 \mathrm{bp}$ in the strains that had not undergone gene replacement and $1200 \mathrm{bp}$ in the strains that contained a truncated form of hasP (Supplementary Fig. 1B). DNA sequencing of PCR products corresponding to truncated forms of $a s n O$ and has $P$ was also carried out, confirming that an in-frame deletion of $549 \mathrm{bp}$ of the $a s n O$ and 618 bp of the hasP genes had occurred (data not shown). Two S. coelicolor 2377- $\Delta a s n O$ strains and four S. coelicolor MT1110$\Delta a s n O$ strains were generated. In addition three S. coelicolor $2377-$ $\Delta$ has $P$ and S. coelicolor MT1110- $\Delta$ hasP strains were also generated.

CDA production, extraction and analysis. The MT1110 parent, $\triangle a s n O$ and $\Delta$ has $P$ mutant strains were grown for 6 days at $30^{\circ} \mathrm{C}$ in SV2 liquid medium (Hojati et al., 2002). S. coelicolor 2377 parent, $\triangle a s n O$ and $\Delta$ has $P$ mutant strains were grown for 6 days at $30^{\circ} \mathrm{C}$ in SM22 liquid medium (Hojati et al., 2002). The culture supernatants were acidified to $\mathrm{pH} 2$ with $0.5 \mathrm{M} \mathrm{HCl}$ and passed through Varian MegaBond Elute SPE cartridges (Varian) as described previously (Hojati et al., 2002). The CDA extracts were then eluted with $40 \%$ acetonitrile in $\mathrm{H}_{2} \mathrm{O}$ and dried under reduced pressure. Liquid chromatography (LC)-MS analysis of the crude extracts was carried out on a Micromass LCT orthogonal acceleration time of flight mass spectrometer equipped with an electrospray ionization source run in positive mode, combined with a Waters 2790 separation module. Gradient elution was carried out on a Luna C18 $150 \times 4.6 \mathrm{~mm} 3 \mu \mathrm{m}$ analytical column (Phenomenex). Solvent A was $0.1 \%$ formic acid and solvent B was acetonitrile/ $0.1 \%$ formic acid. A gradient of $20 \%$ $\mathrm{B}$ rising to $70 \%$ over $10 \mathrm{~min}$, followed by $100 \%$ B held for $5 \mathrm{~min}$ at a flow rate of $1 \mathrm{ml} \mathrm{min}{ }^{-1}$ was applied.

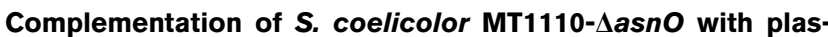
mid-borne asno. To construct a Streptomyces expression plasmid bearing asn $\mathrm{O}$, the gene sequence was PCR-amplified from cosmid ScE29 with primers $29.05 \mathrm{~F} 2$ and 29.05R2, containing restriction sites for $\mathrm{XhoI}$ and NdeI, respectively. The purified product was restricted and ligated into E. coli expression vector pET-15b, resulting in the 6708 bp plasmid pJN14. The asnO gene was excised from pJN14 by digestion with $\mathrm{BamHI}$ and $\mathrm{XhoI}$, and ligated into restriction-digested pIJ6021 (Takano et al., 1995), resulting in the 8904 bp plasmid pJN15. The ligation products were used to transform Streptomyces lividans 1326, and positive transformants were selected by growth on medium containing kanamycin. pJN15 was isolated from transformants, checked by restriction analysis, and introduced into the $S$. coelicolor MT1110- $\Delta a s n O$ mutant by protoplast transformation. The plasmid-complemented mutant was cultivated in SV2 medium supplemented with kanamycin, and thiostrepton was added at a concentration of $10 \mu \mathrm{g} \mathrm{ml}^{-1}$ to induce expression of AsnO. CDA was extracted from the culture supernatant and analysed by LC-MS, as described above.

Cell-based bioassay of Asn-containing CDAs. Spores of S. coelicolor parent and $\Delta a s n O$ mutant strains were used to inoculate nutrient agar plates in the presence and absence of $\mathrm{Ca}^{2+}(12 \mathrm{mM})$. After 2 days' incubation at $30^{\circ} \mathrm{C}$, an overnight culture of the CDAsensitive Bacillus mycoides indicator strain was used to overlay the plates in soft nutrient agar (per litre: $13 \mathrm{~g}$ nutrient broth, $7 \mathrm{~g}$ Bactoagar). Calcium-dependent bioactivity was observed as a zone of inhibition after one night of incubation at $30^{\circ} \mathrm{C}$ (Fig. 5A).

Production and isolation of CDA6a from 2377- $\Delta a s n O$. Sterile SM22 liquid medium (2 1) was inoculated with S. coelicolor 2377$\Delta a s n O$ and then incubated at $28^{\circ} \mathrm{C}$ and 180 r.p.m. for 5 days. The cells were removed by centrifugation and the CDA6a in the resulting supernatants was extracted onto Varian C-18 Bond Elute SPE cartridges (2 g), as described previously (Hojati et al., 2002). Purification of the crude extracts was achieved by preparative and semi-preparative reversed-phase HPLC: Phenomenex C-18 $10 \mu \mathrm{m}$, $250 \times 21.2 \mathrm{~mm}$ and $5 \mu \mathrm{m}, 250 \times 10 \mathrm{~mm}$ columns; solvent $\mathrm{A} \mathrm{H}_{2} \mathrm{O}$ with $0.1 \% \mathrm{HCO}_{2} \mathrm{H}$; solvent $\mathrm{B}$ acetonitrile with $0.1 \% \mathrm{HCO}_{2} \mathrm{H}$. The flow rates were 20 and $5 \mathrm{ml} \mathrm{min}{ }^{-1}$, respectively, with a starting gradient of $0 \% \mathrm{~B}$ and $100 \% \mathrm{~A}$, increasing to $100 \% \mathrm{~B}$ over $30 \mathrm{~min}$ and then held for a further $5 \mathrm{~min}$. Fractions for the peak containing mainly CDA6a (retention time $\sim 17.3 \mathrm{~min}$ ) were pooled and evaporated under reduced pressure to give CDA6a (1.0 mg, 90\% pure).

Well-based bioassay of CDA6a. CDA-sensitive strain Micrococcus luteus was grown overnight at $30^{\circ} \mathrm{C}$ in Oxoid nutrient broth $(5 \mathrm{ml})$. This culture was used to seed Oxoid nutrient agar plates in the presence or absence of added $\mathrm{Ca}^{2+}(16 \mathrm{mM})$. Agar plugs were cut from the dried plates, creating wells into which a sterile aqueous solution of CDA6a $(\sim 100 \mu \mathrm{g}$ in $100 \mu \mathrm{l})$ was added. The plates were then incubated overnight at $30^{\circ} \mathrm{C}$ (Fig. 5B).

\section{RESULTS}

\section{AsnO and HasP sequence analysis}

BLAST sequence similarity searches on the SCO3236 (asnO) and SCO3234 (has $P$ ) gene products were performed. In the case of HasP, no recently deposited protein sequences, in addition to the aminoglycoside phosphotransferases noted earlier (Hojati et al., 2002), were identified which might provide insight into the function of the putative phosphotransferase. On the other hand, several new sequences, in addition to CAS, were identified which exhibit high similarity to the putative asparagine oxygenase (AsnO). For example, LptL possesses $45 \%$ identity across 310 out of 330 amino acid residues, and is predicted to be involved in the hydroxylation of the Asn residue at position 3 of A54145 in Streptomyces fradiae (Miao et al., 2006). In addition Lenduracididine hydroxylase, VioC (Yin \& Zabriskie, 2004; Ju et al., 2004), exhibits $33 \%$ identity across a 292 amino acid alignment with AsnO, and the L-arginine hydroxylase MppO (Haltli et al., 2005) possesses $31 \%$ identity across 300 amino acid residues. All of these proteins possess the His-1 (HXE) and the C-terminal His-3 (DNXXXXH) motifs which make up the $\mathrm{Fe}(\mathrm{II})$ coordination sphere, which supports their known or putative function as $\mathrm{Fe}(\mathrm{II}) / 2$ oxoglutarate-dependent oxygenase enzymes (Haltli et al., 2005; Khaleeli et al., 2000).

\section{Generation of S. coelicolor $\Delta a s n O$ and $\Delta$ hasP mutants}

In order to establish the role of the $a s n O$ and has $P$ gene products, both genes were separately deleted from the $S$. coelicolor chromosome using a standard double-crossover 
recombination approach (Fig. 2) (Kieser et al., 2000). The successful generation of the required in-frame mutants was confirmed by PCR amplification across the truncated region (Supplementary Fig. S1), followed by DNA sequencing. Knockout experiments were performed in both 2377 and MT1110 strains, since these strains have different growthmedia requirements and produce different profiles of CDAs. The combined information can thus provide valuable complementary information which can assist in validating the results of the separate experiments.

\section{Analysis of S. coelicolor $\Delta$ hasP and $\Delta a s n O$}

The S. coelicolor 2377 parent strains and 2377- $\Delta$ hasP mutant strains were grown in liquid culture, using media and conditions which we have previously shown (Hojati et al., 2002) result in the production of phosphorylated CDAs. The culture supernatants were analysed by LC-MS, which confirmed the previous observation (Hojati et al., 2002) that the parent strain 2377 produces D-3-phosphohydroxyasparagine-containing peptide $\mathrm{CDA} 2 \mathrm{~b}$ as the major product, along with a minor amount of D-3-hydroxyasparagine variants CDA4b and CDA3b (Fig. 3A). In contrast, the 2377- $\Delta$ has $P$ mutants produced only the non-phosphorylated variants $\mathrm{CDA} 3 \mathrm{~b}$ and $\mathrm{CDA} 4 \mathrm{~b}$ under identical growth conditions (Fig. 3B). Indeed, repeated fermentation and analysis of several 2377- $\Delta$ has $P$ mutant strains clearly revealed a complete absence of CDA2b, or any other phosphorylated CDAs. This supports the hypothesis that the gene product HasP is the CDA 3-hydroxyasparaginyl

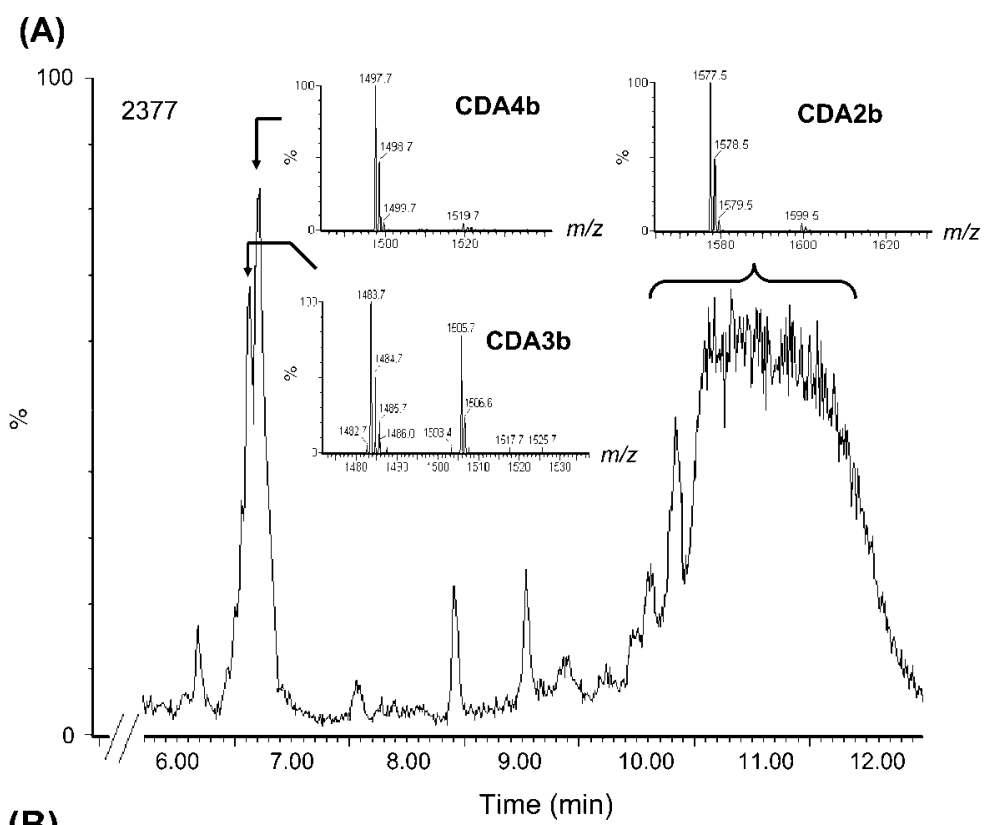

(B)

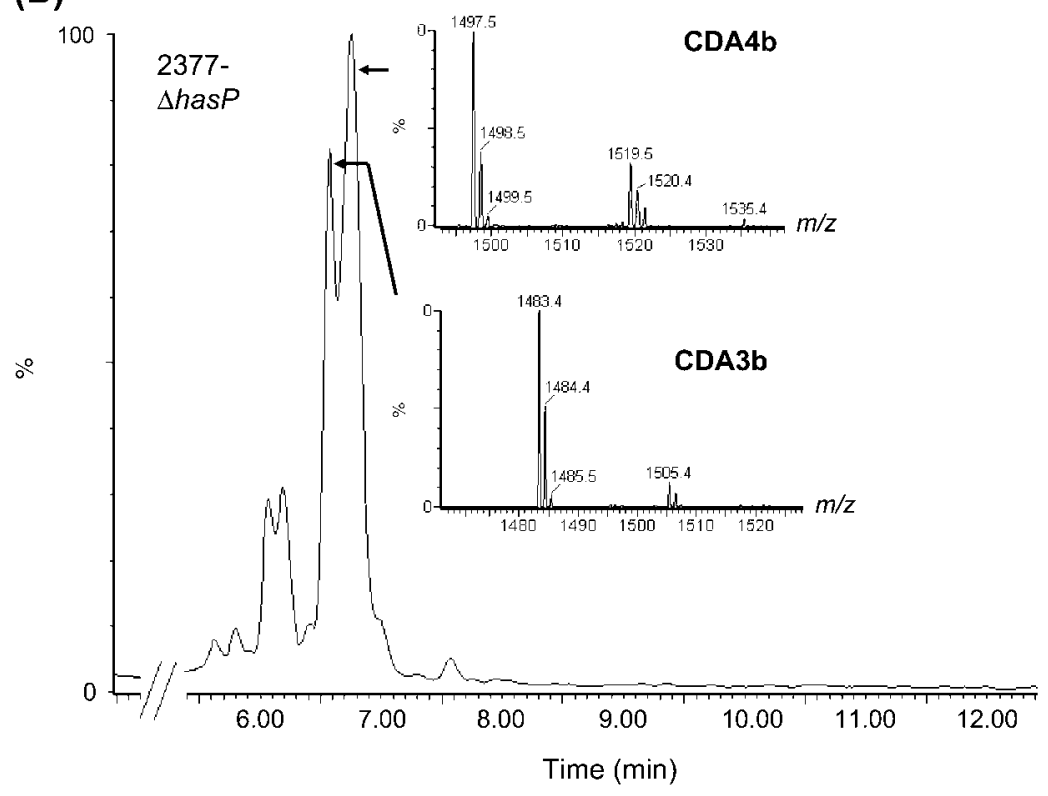

Fig. 3. LC-MS analysis of $S$. coelicolor 2377 parent and 2377- $\Delta$ has $P$ mutant strains. (A) S. coelicolor 2377 parent strain shows CDA2b $\left([\mathrm{M}+\mathrm{H}]^{+}\right.$found 1577.5, requires 1577.5; $[\mathrm{M}+\mathrm{Na}]^{+}$found 1599.5, requires 1599.5), CDA4b $\left([\mathrm{M}+\mathrm{H}]^{+}\right.$found 1497.7, requires 1497.5; $[\mathrm{M}+\mathrm{Na}]^{+}$found 1519.7, requires 1519.5), CDA3b $\left([\mathrm{M}+\mathrm{H}]^{+}\right.$ found 1483.7, requires 1483.5; $[\mathrm{M}+\mathrm{Na}]^{+}$ found 1505.7, requires 1505.5). The LC-MS of CDA2b standards also gave a very broad peak, with a retention time $\left(R_{\mathrm{t}}\right)$ of 10.5-12.0 min. This was due to the multiple ionization states of the phosphorylated peptide and the fact that it was not possible to use a buffered eluant, because this interferes severely with mass detection on LCESI-MS. (B) S. coelicolor 2377- $\Delta$ has $P$. Production of phosphorylated CDA2b is completely abolished in this mutant, which produces only CDA4b $\left([\mathrm{M}+\mathrm{H}]^{+}\right.$found $1497.5 ; \quad[\mathrm{M}+\mathrm{Na}]^{+}$found 1519.5) and CDA3b $\left([\mathrm{M}+\mathrm{H}]^{+}\right.$found $1483.4 ;[\mathrm{M}+\mathrm{Na}]^{+}$ found 1505.4). \%, percentage of maximum intensity peak. 
phosphotransferase. Analysis of the parent strain MT1110, grown on a variety of different media, showed only D-3hydroxyasparagine-containing CDAs and failed to reveal any phosphorylated CDAs. Comparison of the phenotype change between MT1110 and MT1110- 4 hasP strains was therefore not possible in this case.

S. coelicolor MT1110 and MT1110- $\Delta a s n O$ strains were grown in liquid media under identical conditions. LCelectrospray ionization (ESI)-MS analysis of MT1110 revealed the 3-MeGlu-containing peptide CDA4a as the major product along with a minor amount of the Glucontaining CDA3a (Fig. 4A). The mutant MT1110- $\Delta a s n O$, however, does not produce any CDA that is common to the MT1110 or 2377 strains (Hojati et al., 2002; Kempter et al., 1997), but instead exhibits a major new product with a retention time of $6.90 \mathrm{~min}$, which exhibited protonated, sodiated and potassiated $\left([\mathrm{M}+\mathrm{H}]^{+},[\mathrm{M}+\mathrm{Na}]^{+}\right.$and $[\mathrm{M}+\mathrm{K}]^{+}$) molecular ions in the ESI-MS consistent molecular weight $(m w)$ 1478.5 Da (Fig. 4B). This CDA variant was therefore assigned as a non-hydroxylated Asncontaining variant, CDA6a (Fig. 1), which is related to CDA4a by the loss of the oxygen (1494.5-16.0 Da). Similarly, the S. coelicolor 2377 parent and 2377- $\Delta a s n O$ mutant strains were grown in liquid culture. As above, the parent strain 2377 produced CDA2b as the major product with a minor amount of CDA4b and $3 \mathrm{~b}$. In contrast, the mutant 2377-_asnO produced none of the previously characterized CDAs. Instead, two new CDA products were identified. The faster-eluting compound, with an LC retention time of $6.90 \mathrm{~min}$, exhibited protonated, sodiated and potassiated molecular ions in the ESI-MS consistent with CDA6a. In addition, a slower-eluting variant $\left(R_{\mathrm{t}}=6.79\right)$ was observed with molecular ions that indicate $m w 1464.5$ (Fig. 4C). This product corresponds to a nonhydroxylated variant of CDA3a (1480.5-16.0 Da), which we have assigned as CDA5a (Fig. 1).

It is thus clear from these complementary results that deletion of $a s n O$ abolishes the normal CDA production profile of 3-OHAsn- and 3-OPAsn-containing peptides, and instead results in the production of two previously uncharacterized Asn-containing CDA variants (designated CDA5a and 6a).

\section{Expression of plasmid-borne asno in the MT1110-AasnO mutant}

In order to gain further confirmation that $\mathrm{AsnO}$ is responsible for the hydroxylation of the Asn residue in CDA, the MT1110- $\Delta a s n O$ strain was complemented by transformation with a thiostrepton-inducible asnO expression vector derived from the Streptomyces plasmid pIJ6021. The plasmid containing MT1110-_asnO mutants was grown on media containing the inducer thiostrepton. LC-MS analysis of the extracts revealed the same CDA profile as that of the MT1110 parent strain, with the production of 3-OHAsn-containing CDA4a and CDA3a being re-established. The results of the asnO knockout and complementation experiments are thus entirely consistent with the AsnO, a single polypeptide, functioning as an asparaginyl oxygenase.

\section{Bioactivity of Asn CDAs}

Parent and $\Delta a s n O$ mutant strains of S. coelicolor 2377 and MT1110 were each separately incubated with and without $\mathrm{Ca}^{2+}$, and overlaid with the CDA-sensitive strain $B$. mycoides. A calcium-dependent zone of inhibition was seen around the two S. coelicolor 2377-_asnO strains, indicating that Asn-containing CDAs retained calciumdependent antimicrobial activity (Fig. 5A). Similarly, all four of the S. coelicolor MT1110- $\triangle a s n O$ strains exhibited calcium-dependent antibiosis with B. mycoides (data not shown). To further confirm that the bioactivity resulted from Asn-containing CDAs, 2377- $\triangle a s n O$ was cultivated on a large scale (2 l), and the resulting CDA6a was purified from the culture supernatant using preparative and semipreparative HPLC. High-resolution ESI-MS $(\mathrm{m} / \mathrm{z}$ 1479.5333, $\mathrm{C}_{67} \mathrm{H}_{79} \mathrm{~N}_{14} \mathrm{O}_{25}$ requires 1479.5340) and UV spectroscopy $\left(\lambda_{\max } 347 \mathrm{~nm}\right.$ in $\left.\mathrm{H}_{2} \mathrm{O}\right)$ characteristic of $\mathrm{Z}$ dehydrotryptophan-containing a-series CDA (Hojati et al., 2002) further confirmed the structure of CDA6a. Well-plate bioassays of CDA6a with $M$. luteus as the indicator stain showed significant calcium-dependent zones of inhibition (Fig. 5B). Given that only $\sim 1 \mathrm{mg}$ of CDA6a could be purified from a 21 culture of $S$. coelicolor $2377-\Delta a s n O$, it was not practical to obtain sufficient quantities of CDA6a to determine accurate MIC values, for comparison with the other known CDAs. Nevertheless, from the size of the zones of inhibition and the concentrations of CDAs used, it is possible to conclude that CDA6a is less active than the corresponding 3-OHAsn-containing CDA4a and CDA3a (Milne et al., 2006).

\section{DISCUSSION}

This work clearly shows that $\mathrm{AsnO}$ and HasP are involved in the biosynthesis of 3-OHAsn and 3-OPAsn in CDA. However, the results do not indicate directly whether the hydroxylation and phosphorylation reactions take place before, during or after peptide assembly. Despite this, clues about the true nature of the substrates for AsnO and HasP can be derived by analysing the amino acid specificity conferring code of the module 9 adenylation domain (A domain) of the nonribosomal peptide synthetase (cdaPS2) responsible for activating Asn or its derivatives. Indeed, the 10 essential active site residues of the module 9 A domain (DLTKIGEVGK) within CdaPS2 have similarity to many other Asn activating A domains from different sources (Stachelhaus et al., 1999; Challis et al., 2000). This indicates that the module 9 A domain will be able to activate Asn, and indeed this must necessarily be the case given the production of Asn-containing lipopeptides CDA5a and 6a in the $\triangle a s n O$ mutants. Furthermore, the module $9 \mathrm{~A}$ domain might possess broad substrate specificity, allowing the activation of 3-OHAsn, which is only slightly larger than Asn. On the 
other hand, it is unlikely that the A domain could activate both 3-OPAsn and Asn, given the very large difference in molecular volume and polarity of these two substrates. Furthermore, we have shown that it is possible to change the active site of the Asp-7 A domain (at the positions underlined: DLTKIGAVNK) to match exactly the sequence of the module 9 (Asn) $\bar{A}$ domain (DLTKIGEVGK). The resulting mutant produces a new lipopeptide product, CDA-7N, with Asn at position 7 instead of Asp (Uguru et al., 2004). Conceivably, if free Asn was hydroxylated to 3OHAsn prior to A-domain activation, then this experiment could have resulted in CDAs with 3-OHAsn or 3-OPAsn at position 7 , which was not the case. However, the upstream condensation domain of module 7 was unaltered, and thus retained specificity for the Asp-S-PCP precursor. Thus, the condensation of any 3-OHAsn-S-PCP intermediate (which may have accumulated) with the upstream hexapeptidyl intermediate would have been severely impaired. Indeed, this could account for the fact that the Asp-7 A domain double point mutant produces considerably more of the hydrolysed CDA hexapeptide intermediate than the Asncontaining CDA-7N (Uguru et al., 2004).
Whilst the results presented here and previously (Hojati et al., 2002; Uguru et al., 2004) do not indicate the timing of the Asn hydroxylation, the evidence suggests that phosphorylation is most likely to occur after A-domain activation. Therefore, 3-OHAsn-containing CDAs (e.g. CDA3a, 3b, 4a and $4 \mathrm{~b}$ ) are the most likely substrates for HasP. Alternatively, the possibility that phosphorylation occurs during peptide assembly cannot be ruled out, as other nonribosomal peptide-tailoring enzymes are suggested to modify peptidyl-thioester intermediates tethered to PCP domains. Indeed, there is evidence that oxidative coupling reactions between the phenolic side chains most likely occur on the peptidyl-thioester intermediates during the assembly of vancomycin and related glycopeptides (Zerbe et al., 2004; Bischoff et al., 2005).

It is also interesting to note that Asn-containing CDAs retain calcium-dependent antimicrobial activity (Fig. 5) and that 3-OHAsn or 3-OPAsn motifs are therefore not essential for calcium binding. Interestingly, the 3-OHAsn/Asp residues found in EGF are suggested to be involved in calcium binding (Valcarce et al., 1999; Lancaster et al., 2004). Thus,
(A)
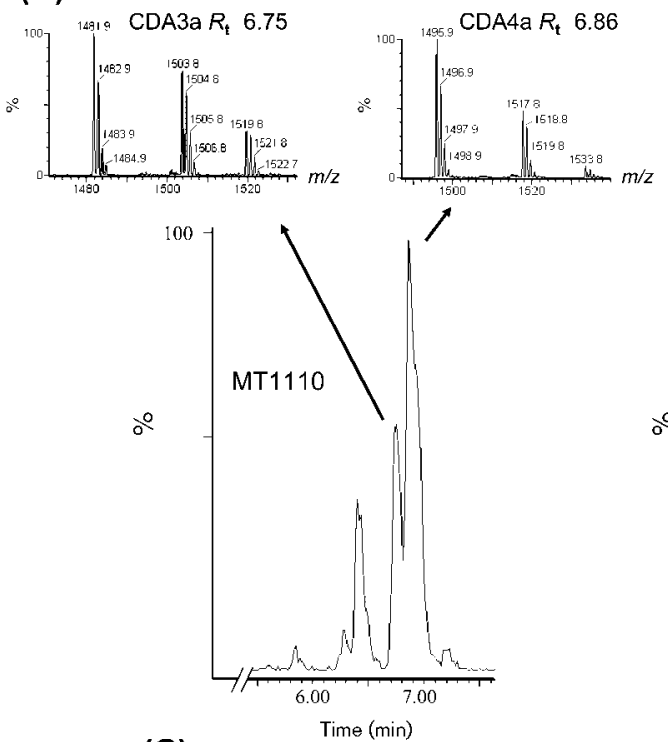

(C)

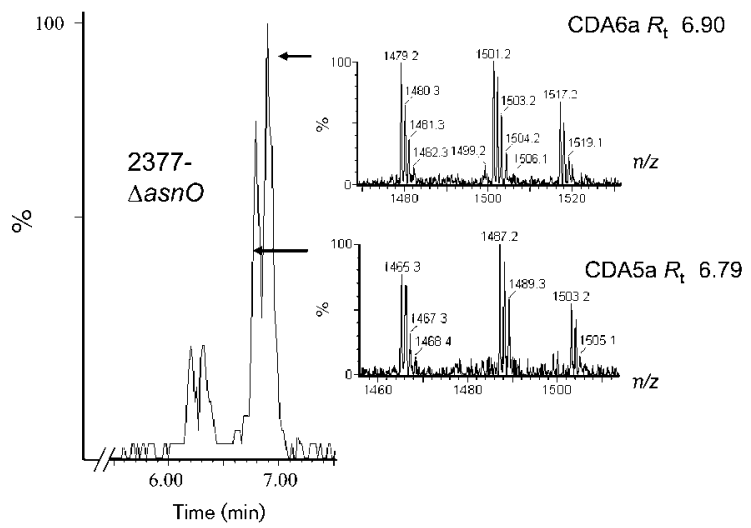

(B)
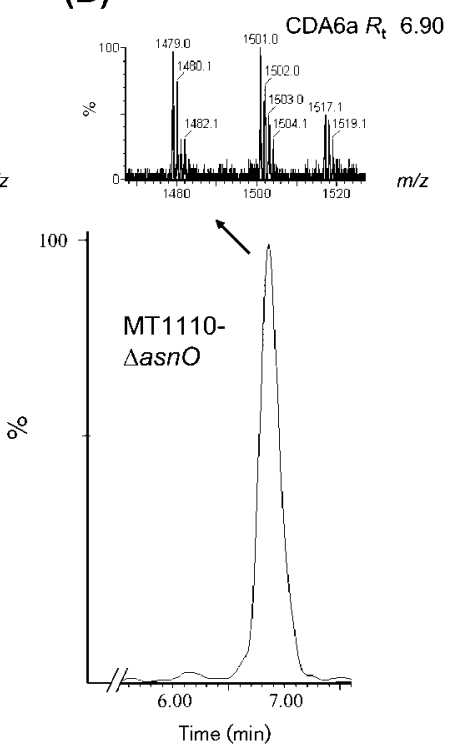

CDA6a $R_{\mathrm{t}} 6.90$
Fig. 4. LC-MS analysis of MT1110 and $\Delta$ asnO mutants. (A) S. coelicolor MT1110 produces CDA3a $\left([\mathrm{M}+\mathrm{H}]^{+}\right.$found 1481.9, requires $1481.5 ;[\mathrm{M}+\mathrm{Na}]^{+}$found 1503.8 , requires 1503.5; $[\mathrm{M}+\mathrm{K}]^{+}$found 1519.8 , requires 1519.5), CDA4a $\left([\mathrm{M}+\mathrm{H}]^{+}\right.$found 1495.9, requires $1495.5 ;[\mathrm{M}+\mathrm{Na}]^{+}$found 1517.8, requires 1517.5; $[\mathrm{M}+\mathrm{K}]^{+}$found 1533.8, requires 1533.5). (B) $S$. coelicolor MT1110- $\Delta$ asnO produces CDA6a $\left([\mathrm{M}+\mathrm{H}]^{+}\right.$ found 1479.0 , requires $1479.5 ;[\mathrm{M}+\mathrm{Na}]^{+}$ found 1501.0, requires 1501.5; $[\mathrm{M}+\mathrm{K}]^{+}$ found 1517.1, requires 1517.5). (C) S. coelicolor 2377- $\triangle$ asnO produces CDA5a $\left([\mathrm{M}+\mathrm{H}]^{+}\right.$found 1465.3, requires 1465.5; $[\mathrm{M}+\mathrm{Na}]^{+}$found 1487.2 , requires 1487.5; $[\mathrm{M}+\mathrm{K}]^{+}$found 1503.2, requires 1503.5) and CDA6a $\left([\mathrm{M}+\mathrm{H}]^{+}\right.$found 1479.2; $[\mathrm{M}+\mathrm{Na}]^{+}$found 1501.2; $[\mathrm{M}+\mathrm{K}]^{+}$found 1517.2). $R_{\mathrm{t}}=$ retention time (min). \%, percentage of maximum intensity peak. 


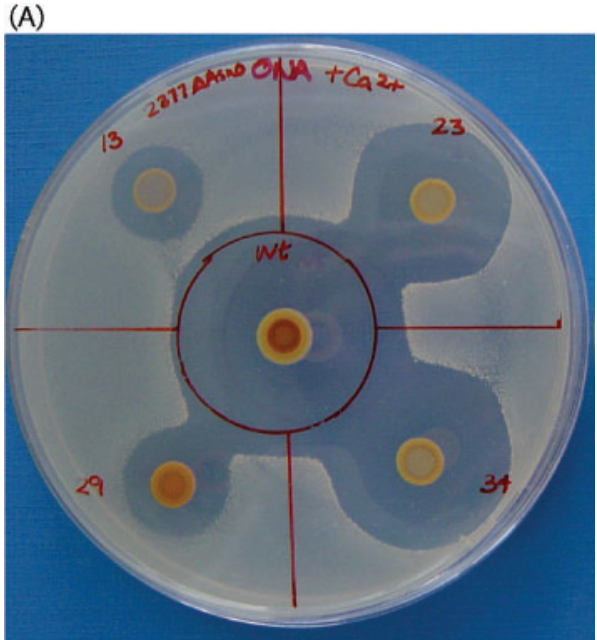

(B)

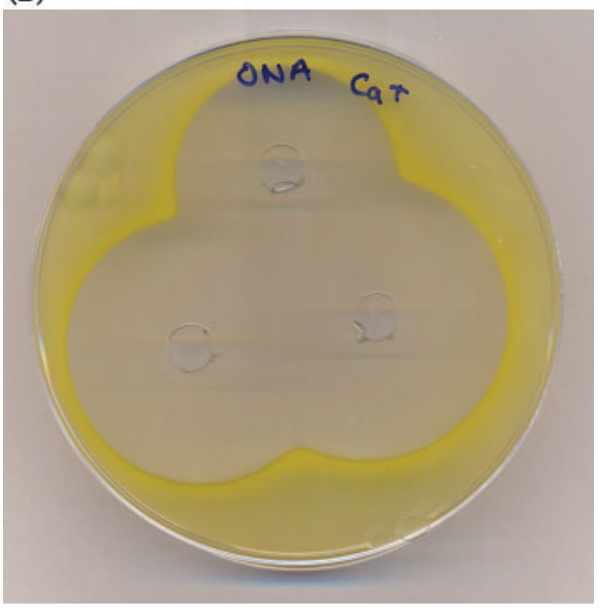

Fig. 5. Bioactivity of Asn-containing CDAs. (A) Cell bioassay of 2377- $\Delta a s n O$ strains overlaid with $B$. mycoides as the indicator strain in the presence of $\mathrm{Ca}^{2+}(12 \mathrm{mmol})$. Strains 13 and 29 are $\Delta$ asnO mutant strains, whilst strains 23 and 34 had been transformed with the $\Delta a s n O$ deletion construct, but proved not to possess the asn $\mathrm{O}$ deletion and therefore retained the same phenotype as the parent strain 2377. The parent 2377 strain is shown in the centre of the plate. No zones of inhibition were present in the control plates which contained no $\mathrm{Ca}^{2+}$. (B) Well-plate bioassay with purified CDA6a (100 $\mu \mathrm{g}$ in $100 \mu \mathrm{l})$ and $M$. luteus as the indicator strain, in the presence of $\mathrm{Ca}^{2+}(16 \mathrm{mM})$. Again, zones of inhibition were completely absent in the control plates which possessed no $\mathrm{Ca}^{2+}$.

whilst our results indicate that 3-OHAsn residues are not essential for coordination of $\mathrm{Ca}^{2+}$ ions to $\mathrm{CDA}$, the possibility that $\beta$-hydroxylation of Asn in nonribosomal as well as ribosomal peptides is evolutionarily linked to the propensity of the $\beta$-hydroxylated peptide products to bind $\mathrm{Ca}^{2+}$ ions cannot be discounted (Lancaster et al., 2004). In addition to this, it is also worth noting that the S. coelicolor

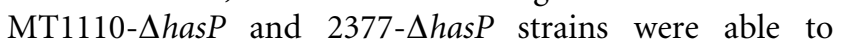
produce non-phosphorylated CDAs and maintain growth on media supplemented with $\mathrm{Ca}^{2+}$. This suggests that HasP is not part of the host self-resistance mechanism, as is the case for other phosphotransferases which share sequence similarity (Lyutzkanova et al., 1997).

\section{ACKNOWLEDGEMENTS}

This work was supported by the Biotechnology and Biological Sciences Research Council (BBSRC) through research grants (36/B12126 and BB/C503662) and through PhD studentships to L. G., C. M. and A. P., and by the Engineering and Physical Sciences Research Council (EPSRC) through the award of a PhD studentship to J. M. N. L. G. and C. M. thank GlaxoSmithKline, and A. P. thanks Biotica Technology Ltd, for CASE awards.

\section{REFERENCES}

Baldwin, J. E., Lloyd, M. D., Wha-Son, B., Schofield, C. J., Elson, S. W., Baggaley, K. H. \& Nicholson, N. H. (1993). A substrate analogue study on clavaminic acid synthase: possible clues to the biosynthetic origin of proclavamic acid. J Chem Soc Chem Commun500-502.

Baltz, R. H., Miao, V. \& Wrigley, S. K. (2005). Natural products to drugs: daptomycin and related lipopeptide antibiotics. Nat Prod Rep 22, 717-741.

Bierman, M., Logan, R., O'Brien, K., Seno, E. T., Nagaraja Rao, R. \& Schoner, B. E. (1992). Plasmid cloning vectors for the conjugal transfer of DNA from Escherichia coli to Streptomyces spp. Gene 116, 43-49.

Birnboim, H. C. \& Doly, J. (1979). A rapid alkaline extraction procedure for screening recombinant plasmid DNA. Nucleic Acids Res 7, 1513-1523.

Bischoff, D., Bister, B., Bertazzo, M., Pfeifer, V., Stegmann, E., Nicholson, G. J., Keller, S., Pelzer, S., Wohlleben, W. \& Sussmuth, R. D. (2005). The biosynthesis of vancomycin-type glycopeptide antibiotics: a model for oxidative side-chain cross-linking by oxygenases coupled to the action of peptide synthetases. Chembiochem 6, 267-272.

Challis, G. L., Ravel, J. \& Townsend, C. A. (2000). Predictive, structure-based model of amino acid recognition by nonribosomal peptide synthetase adenylation domains. Chem Biol 7, 211-224.

Chen, H., Thomas, M. G., O'Connor, S. E., Hubbard, B. K., Burkart, M. D. \& Walsh, C. T. (2001). Aminoacyl-S-enzyme intermediates in $\beta$ hydroxylation and $\alpha, \beta$-desaturation of amino acids in peptide antibiotics. Biochemistry 40, 11651-11659.

Chen, H., Hubbard, B. K., O'Connor, S. E. \& Walsh, C. T. (2002). Formation of $\beta$-hydroxyhistidine in the biosynthesis of nikkomycin antibiotics. Chem Biol 9, 103-112.

Fukuda, D. S., Du Bus, R. H., Baker, P. J., Berry, D. M. \& Mynderse, J. S. (1990). A54145, a new lipopeptide antibiotic complex: isolation and characterization. J Antibiot 43, 594-600.

Haltli, B., Tan, Y., Magarvey, N. A., Wagenaar, M., Yin, X., Greenstein, M., Hucul, J. A. \& Zabriskie, T. M. (2005). Investigating $\beta$-hydroxyenduracididine formation in the biosynthesis of the mannopeptimycins. Chem Biol 12, 1163-1168.

Hewitson, K. S., McNeill, L. A., Riordan, M. V., Tian, Y. M., Bullock, A. N., Welford, R. W., Elkins, J. M., Oldham, N. J., Bhattacharya, S. \& other authors (2002). Hypoxia-inducible factor (HIF) asparagine hydroxylase is identical to factor inhibiting HIF (FIH) and is related to the cupin structural family. J Biol Chem 277, 26351-26355. 
Hindle, Z. \& Smith, C. P. (1994). Substrate induction and catabolite repression of the Streptomyces coelicolor glycerol operon are mediated by the GlyR protein. Mol Microbiol 12, 737-745.

Hojati, Z., Milne, C., Harvey, B., Gordon, L., Borg, M., Flett, F., Wilkinson, B., Sidebottom, P. J., Rudd, B. A. M. \& other authors (2002). Structure, biosynthetic origin and engineered biosynthesis of calcium-dependent antibiotics from Streptomyces coelicolor. Chem Biol 9, 1175-1187.

Hopwood, D. A. \& Wright, H. M. (1983). CDA is a new chromosomally-determined antibiotic from Streptomyces coelicolor A3(2). J Gen Microbiol 129, 3575-3579.

Jia, S., McGinnis, K., VanDusen, W. J., Burke, C. J., Kuo, A., Griffin, P. R., Sardana, M. K., Elliston, K. O., Stern, A. M. \& Friedman, P. A. (1994). A fully active catalytic domain of bovine aspartyl (asparaginyl) $\beta$-hydroxylase expressed in Escherichia coli: characterization and evidence for the identification of an active-site region in vertebrate $\alpha$-ketoglutarate-dependent dioxygenases. Proc Natl Acad Sci U S A 91, 7227-7231.

Ju, J., Ozanick, S. G., Shen, B. \& Thomas, M. G. (2004). Conversion of $(2 S)$-arginine to $(2 S, 3 R)$-capreomycidine by VioC and VioD from the viomycin biosynthetic pathway of Streptomyces sp. strain ATCC11861. Chembiochem 5, 1281-1285.

Kato, T., Hinoo, H., Terui, Y., Kikuchi, J. \& Shoji, J. (1988). The structures of katanosins A and B. J Antibiot 41, 719-725.

Kempter, C., Kaiser, D., Haag, S., Nicholson, G., Gnau, V., Walk, T., Gierling, G. H., Decker, H., Zahner, H. \& other authors (1997). CDA: calcium-dependent peptide antibiotics from Streptomyces coelicolor A3(2) containing unusual residues. Angew Chem Int Ed Engl 36, 498-501.

Khaleeli, N., Busby, R. W. \& Townsend, C. A. (2000). Site-directed mutagenesis and biochemical analysis of the endogenous ligands in the ferrous active site of clavaminate synthase. The His-3 variant of the 2-His-1-carboxylate model. Biochemistry 39, 8666-8673.

Kieser, T., Bibb, M. J., Buttner, M. J., Chater, K. F. \& Hopwood, D. A. (2000). Practical Streptomyces Genetics. Norwich, UK: John Innes Foundation.

Lancaster, D. E., McDonough, M. A. \& Schofield, C. J. (2004). Factor inhibiting hypoxia-inducible factor (FIH) and other asparaginyl hydroxylases. Biochem Soc Trans 32, 943-945.

Lu, W., Oberthur, M., Leimkuhler, C., Tao, J., Kahne, D. \& Walsh, C. T. (2004). Characterization of a regiospecific epivancosaminyl transferase GtfA and enzymatic reconstitution of the antibiotic chloroeremomycin. Proc Natl Acad Sci U S A 101, 4390-4395.

Lyutzkanova, D., Distler, J. \& Altenbuchner, J. (1997). A spectinomycin resistance determinant from the spectinomycin producer Streptomyces flavopersicus. Microbiology 143, 2135-2143.

McCafferty, D. G., Cudic, P., Frankel, B. A., Barkallah, S., Kruger, R. G. \& Li, W. (2002). Chemistry and biology of the ramoplanin family of peptide antibiotics. Peptide Science 66, 261-284.

Miao, V., Coëffet-LeGal, M.-F., Brian, P., Brost, R., Penn, J., Whiting, A., Martin, S., Ford, R., Parr, I. \& other authors (2005). Daptomycin biosynthesis in Streptomyces roseosporus: cloning and analysis of the gene cluster and revision of peptide stereochemistry. Microbiology 151, $1507-1523$
Miao, V., Brost, R., Chapple, J., She, K., Coëffet-Le Gal, M.-F. \& Baltz, R. H. (2006). The lipopeptide antibiotic A54145 biosynthetic gene cluster from Streptomyces fradiae. J Ind Microbiol Biotechnol 33, 66-74.

Milne, C., Powell, A., Jim, J., Al Nakeeb, M., Smith, C. P. \& Micklefield, J. (2006). Biosynthesis of the (2S, 3R)-3-methyl glutamate residue of nonribosomal lipopeptides. J Am Chem Soc 128, 11250-11259.

Oh, S. H. \& Chater, K. F. (1997). Denaturation of circular or linear DNA facilitates targeted integrative transformation of Streptomyces coelicolor A3(2): possible relevance to other organisms. J Bacteriol 17, 122-127.

Paget, M. S., Hintermann, G. \& Smith, C. P. (1994). Construction and application of streptomycete promoter probe vectors which employ the Streptomyces glaucescens tyrosinase-encoding gene as a reporter. Gene 146, 105-110.

Raja, A., LaBonte, J., Lebbos, J. \& Kirkpatrick, P. (2003). Daptomycin. Nat Rev Drug Discovery 2, 943-944.

Salowe, S. P., Marsh, E. N. \& Townsend, C. A. (1990). Purification and characterization of clavaminate synthase from Streptomyces clavuligerus: an unusual oxidative enzyme in natural product biosynthesis. Biochemistry 29, 6499-6508.

Sambrook, J., Fritsch, E. F. \& Maniatis, T. (2000). Molecular Cloning: a Laboratory Manual, 2nd edn. Cold Spring Harbor, NY: Cold Spring Harbor Laboratory

Stachelhaus, T., Mootz, H. D. \& Marahiel, M. A. (1999). The specificity-conferring code of adenylation domains in nonribosomal peptide synthetases. Chem Biol 6, 493-505.

Takano, E., White, J., Thompson, C. \& Bibb, M. J. (1995). Construction of thiostrepton-inducible, high-copy number expression vectors for Streptomyces spp. Gene 166, 133-137.

Uguru, G. C., Milne, C., Borg, M., Flett, F., Smith, C. P. \& Micklefield, J. (2004). Active-site modifications of adenylation domains lead to hydrolysis of upstream nonribosomal peptidyl thioester intermediates. J Am Chem Soc 126, 5032-5033.

Vértesy, L., Ehlers, E., Kogler, H., Kurz, M., Meiwes, J., Seibert, G., Vogel, M. \& Hammann, P. (2000). Friulimicins: novel lipopetide antibiotics with peptidoglycan synthesis inhibiting activity from Actinoplanes friuliensis sp. nov. J Antibiot 53, 816-827.

Valcarce, C., Björk, I. \& Stenflo, J. (1999). The epidermal growth factor precursor: a calcium-binding, $\beta$-hydroxyasparagine containing modular protein present on the surface of platelets. Eur J Biochem 260, 200-207.

Walker, S., Chen, L., Hu, Y., Rew, Y., Shin, D. \& Boger, D. L. (2005). Chemistry and biology of ramoplanin: a lipoglycodepsipeptide with potent antibiotic activity. Chem Rev 105, 449-476.

Yin, X. \& Zabriskie, T. M. (2004). VioC is a non-heme iron, $\alpha$ ketoglutarate-dependent oxygenase that catalyzes the formation of $3 S$-hydroxy-L-arginine during viomycin biosynthesis. Chembiochem 5, 1274-1277.

Zerbe, K., Woithe, K., Li, D. B., Vitali, F., Bigler, L. \& Robinson, J. A. (2004). An oxidative phenol coupling reaction catalyzed by oxyB, a cytochrome $\mathrm{P} 450$ from the vancomycin-producing microorganism. Angew Chem Int Ed Engl 43, 6709-6713.

Edited by: R. P. Mellado 